\title{
Pharmacological pain management in patients with chronic hepatic disease
}

\author{
László Irsay ${ }^{1}$, Alexandra Checicheș ${ }^{2}$, Dănuț Perja ${ }^{2}$, Ileana Monica Borda ${ }^{1}$, Gabriela Dogaru ${ }^{1}$, \\ Rodica Ungur ${ }^{1}$, Alina Ciubean ${ }^{1}$, Viorela Ciortea ${ }^{1}$
}

Corresponding author: László Irsay, E-mail: irsaylaszlo@gmail.com,

\section{1. "Iuliu Hatieganu" University of Medicine and Pharmacy Cluj-Napoca, Romania} 2. Clinical Rehabilitation Hospital Cluj-Napoca, Romania

\begin{abstract}
The liver has a central role in the pharmacokinetics (absorption, distribution, elimination, etc.) of drugs. With hepatic insufficiency, the metabolism of drugs decreases, which accumulates metabolically or toxic active products. Some medicines can aggravate a pre-existing liver disease. Medicines used in this group of patients (especially diuretics and centrally acting preparations) can often cause impaired renal function or hepatic encephalopathy. The general principles of assessment, pain management and analgesia should be prescribed taking into account the Word Health Organization (WHO) recommendations for these patients, with careful and frequent monitoring of patient progress during treatment administration.
\end{abstract}

Key words: hepatotoxicity, hepatic dysfunction, cirrhosis, pain medication,

\section{Introduction}

Recent decades have led to an increase in the prevalence of chronic illness in general, with the impact being felt at the level of quality of life. Among the liver diseases, pain is one of the most common reasons why patients are addressing medical services, with a frequency ranging from $17-24 \%$ in cases of cirrhosis to $67 \%$ in those with chronic viral hepatitis C (1). Unfortunately, this symptom is poorly understood, difficult to approach and often has inappropriate treatment. Repercussions can be summed up by a change in the quality of life for this group of people (2). Recent data suggests that pain is related to psychiatric symptoms, race and progression of dysfunction rather than to the etiologic factor of hepatic impairment (3).

The primary fear for practitioners is related to the risk of hepatotoxicity and accumulation of toxic metabolites of pain medication, requiring knowledge of pharmacokinetic details along with the profile of their side effects. In principle, for patients with a mild degree of hepatic impairment, the antialgic therapeutic palette is the same as that is used with people who have no liver disorder. Susceptibility to the onset of side effects increases with the impairment of hepatic function, with changes in the pharmacokinetics and hemodynamics being incriminated (4).

There are a number of so-called "classical" tests to evaluate liver function, taken from the blood (serum transaminases, bilirubin, alkaline phosphatase, prothrombin time, albumin), but they do not really appreciate "how well it works" or not.
Good, modified values may have extrahepatic etiology, or values may be within normal limits even if patients experience an advanced stage of hepatic impairment. More targeted tests have been developed to quantify the ability of the liver to transport organic anions or drug metabolism, such as green indocyanine clearance, but use in clinical practice is rare $(5,6)$.

The reluctance to administer antialgic medication in patients with hepatic pathology may be justified in part because of the lack of a clear limit of liver function from which a dose adjustment or choice of a different preparation is required. As a general rule, patients with severe impairment (decompensated fibrosis or cirrhosis) need to modify dosages especially for those with portal hypertension or renal insufficiency. Exceptions include active alcohol or polypragmazia, where severe hepatotoxicity of concomitant paracetamol can occur regardless of the stage of hepatic disease $(7,8)$.

Correlation between liver dysfunction and physical function has not been rigorously investigated. A study has highlighted the presence of a motor deficit at Child-Pugh scores $\geq 6$. It is assumed that the mechanism of appearance resides in poor muscle protein synthesis encountered in liver dysfunctions. The results support the idea that this category of patients has a higher risk of developing sarcopenia, which by extrapolation recommends developing a physiotherapy program taking into account the ChildPugh score (9). 
This article proposes a review of the most used pain medication and anti-rheumatic drugs in patients with coexisting liver disease. In the search we used as liver terms "dysfunction", "failure", "impairment", "insufficiency" and separate hepatic cirrhosis. Most of the existing studies have small sample size, with no placebo group or case studies or expert opinion. In many situations, "cautious use" is found not only in the medicine leaflet, but also in published case studies. The recommendation of the authors is to monitor liver function and to know the pharmacokinetics of the drug. Another important aspect is the pathological context in which antirheumatic pain medications are administered: most patients with liver disease have a series of comorbidities (renal, cardiovascular disease) that prevent or contraindicate the administration of some drugs. Also, co-morbidities require other types of treatments that interfere with antialgic drugs, which further restrain therapeutic benefits. The following list of pain medication takes into account the WHO pain treatment leader (stage I-II-III).

\section{ANALGESICS}

\section{- STAGE I}

\section{PARACETAMOL:}

Because of its good safety profile along with the absence of sedation or nephrotoxicity, it is the pain medication of choice for patients with hepatic impairment under the recommended dosage (4). It should be noted, however, that about half of the overdose cases occur accidentally, given that many over-the-counter products are combined drugs also containing paracetamol (10).

The frequent recommendation for cirrhotic patients with advanced liver disease or alcohol users is not to exceed $2 \mathrm{~g} /$ day $(11,12)$. For those with severe alcoholic liver or acute liver dysfunction, paracetamol should be avoided (10).

\section{NON-STEROIDIC ANTIINFLAMMATORY DRUGS (NSAIDs)}

Their systemic administration is not recommended for patients with cirrhosis or severe chronic liver disease (including aspirin). Topical products may be considered as a therapeutic option. However, more studies are needed on their safety profile (there is some degree of systemic absorption and it is assumed that this would not suppose to increase any risks for those patients with cirrhosis).
These restrictions are explained by several mechanisms: inhibition of prostaglandin production with aggravation of hydrosaline retention and implicit ascites, increased risk of gastrointestinal haemorrhages (varicose and non-varicose) due to thrombocytopenia and precipitation of acute renal insufficiency (approximately half of the patients with cirrhosis present concomitant kidney damage leading to increased likelihood of toxicity) $(3,13)$.

\section{- STAGE II}

The use of opioids is controversial as the liver is the main site for their metabolism. In liver disease, the clearance of drugs can be low and/or because of the decreased metabolism during oral administration the first liver passage can be fast (3). In the case of long term opioid consumption, tolerance should be considered, which implies a dose escalation, implicitly a higher risk for the development of hepatic encephalopathy (4).

\section{CODEINE}

It is a weak opioid, a precursor drug and its analgesic effects being installed only after conversion to morphine via CYP2d6. In hepatic impairment, the intensity of analgesia is diminished or even absent because of the decrease in the oxidation capacity of this enzyme. At the same time, diminished clearance of codeine should be taken into account, which may lead to accumulation of the drug and, implicitly, to depressed ventilation (4). There are, on the other hand, "fast/ultra fast" metabolizers. In these patients, morphine conversion of codeine occurs rapidly, reaching higher plasma concentrations of morphine; they are much more predisposed for developing adverse reactions even for common doses (14).

The recommendation is to choose other therapeutic alternatives (13).

\section{TRAMADOL}

It is a centrally acting synthetic compound that is oxidized at the liver in the active metabolite - Odesmethyltramadol (M1), which is metabolized by CYP2D6, and its antialgic properties are therefore variable (3). Compared with other preparations in its class, tramadol has more blurred effects of sedation or respiratory depression and the risk of developing tolerance is also lower. However, it decreases the seizure threshold and may trigger serotonin syndrome in subjects treated with tricyclic antidepressants or SSRIs $(7,13,15)$. 
In patients with hepatic impairment the preferred forms are those with immediate release. Side effects to prolonged release forms are more capricious and more difficult to manage, with high risk of accumulation.

In cirrhosis, the recommended dose is $50 \mathrm{mg} /$ per os at 12 hours, with an increase in the interval between administrations. Caution should be exercised in patients with depression and those with a history of seizures should be avoided (13).

\section{STAGE III}

\section{MORPHINE}

In advanced liver disease and cirrhosis it was found to have low plasma clearance, increased bioavailability and half-life (3).

It has two main metabolites, one with antalgic activity and the other with neurotoxic (confusion, seizures, respiratory depression) effects. The expert opinions are that people with liver pathology should be administered with cautious morphine, double the time between administrations and using a reduced oral dose. If the patient suffers concomitantly from chronic kidney disease, morphine should not be given (risk of seizures, respiratory distress, and hepatic encephalopathy) (4).

The treatment will be initiated with $5 \mathrm{mg}$ once every 6 hours, orally $(13,16)$.

\section{OXYCODONE}

Due to poor liver metabolism in advanced stages of hepatic impairment, the analgesic potency is highly variable and influenced (3). Half-life is prolonged and there is risk for high plasma concentrations due to high capacity for binding protein.

Should be administered with caution, at low doses and increasing the interval between them $(7,17)$. It's highly recommended to avoid prolonged release forms (13). The recommended dose is $5 \mathrm{mg}$ once every 6 hours, orally (13).

\section{FENTANYL}

It's a synthetic compound of morphine, with superior potency, with strong tropism for proteins, is converted to the liver into inactive and non-toxic compounds (18). It would appear that it could be safely administered to those with minor hepatic impairment, reducing the dosage to those with hypoalbuminemia $(4,13)$.

Applying transdermal patches can only be considered after an inventory of the daily need for short-acting opioids (13).

\section{METADONE}

It is considered to be a safe option for people with severe hepatic impairment or cirrhosis for short period of time despite prolonged half-life. It is important to mention that dose reduction is required in case of hypoalbuminemia $(4,13)$.

\section{ADJUVANT ANALGESICS \\ 1. GABAPENTIN and 2. PREGABALIN}

They are included in the class of anticonvulsants. These drugs are not metabolized in the liver and do not bind to plasma proteins, but their elimination is dependent on kidney function (4). However, case studies have been reported with pregabalin-induced hepatic toxicity (19). Posology is the usual one, with progressive titration.

\section{DEXAMETHAZONE}

In patients with severe hepatic dysfunction, due to increased half-life and hypoalbuminemia an increase in the biological effects has been observed, and numerous side effects are also under discussion (20, $21)$. Dose reduction is required (22).

\section{NORTRIPTILINA}

It is preferable to amitriptyline or imipramine due to the diminished sedation effect and the lower probability of accumulation of its metabolites (13). It presents dose-dependent side effects, mostly anticholinergic and cardiovascular $(7,8,11)$. Treatment will be initiated with $10 \mathrm{mg}$ orally before bedtime, with the possibility of progressively increasing up to $25 \mathrm{mg}(4,13)$.

\section{OTHER COMMON USED DRUGS}

\section{COLCHICINE}

In mild-moderate hepatic impairment: no dose adjustment is required, but patients should be carefully monitored to assess possible adverse effects. In severe hepatic insufficiency: no dose adjustment is required, but a treatment cure should not be repeated more than once every two weeks. For patients requiring repeated treatments, alternative therapy should be considered (23).

\section{ALLOPURINOL}

Hepatic side effects reported were generally mild. Numbers of patients with transaminases level more than 3 times greater than normal were low. During treatment liver function should be monitored. Several cases of hepatotoxicity have been observed in patients treated with allopurinol, and in some patients asymptomatic elevations of alkaline phosphatase or 
serum transaminases have been observed. In patients with pre-existing liver disease, it is recommended to periodically check liver function in the early stages of treatment. In patients with hepatic impairment, it is recommended to reduce allopurinol doses $(21,24)$.

\section{FEBUXOSTAT}

In mild or moderate hepatic dysfunction dose adjustments are not needed. The recommended dose is $80 \mathrm{mg}$ orally every second day $(24,25)$.

Conclusions: Obtaining analgesia in patients with liver disease is a challenge both in terms of the effectiveness of the medication administered and its safety profile. In principle, most pain medication are likely to be useful for this category of patients despite the potential for hepatotoxicity as long as they work with reduced doses and increased intervals between administrations. It is preferable for fast acting drugs and not prolonged release initially with the lowest dose.

\section{Conflicts of interest}

The authors of this paper state that there are no conflicts of interest regarding the study methodology, results and conclusions drawn.

\section{References}

1. Rogal S, Winger D, Bielefeldt K, Szigethy E. Pain and Opioid Use in Chronic Liver Disease. Digestive Diseases and Sciences. 2013 Oct; 58:2976-2985.

2. Megari K. Quality of Life in Chronic Disease Patients. Health Psychol Res. 2013;1(3):e27.

3. Imani F, Motavaf M, Safari S, Alavian SM. The Therapeutic Use of Analgesics in Patients With Liver Cirrhosis: A Literature Review and Evidence-Based Recommendations. Hepat Mon. 2014 October; 14(10): e23539.

4. Hamilton J, Goldberg E, Chopra S. Management of pain in patients with advanced chronic liver disease or cirrhosis. In K. Robson (Ed.), UptoDate. Retrieved March 21, 2019, from https://www.uptodate.com/contents/management -of-pain-in-patients-with-advanced-chronicliver-disease-or-cirrhosis.

5. Newsome PN, Cramb R, Davison SM, et al. Guidelines on the management of abnormal liver blood tests. Gut. 2018 Jan; 67(1):6-19.

6. Sheng QS, Lang R, He Q, Yang YJ, Zhao DF, Chen DZ. Indocyanine green clearance test and model for end-stage liver disease score of patients with liver cirrhosis. Hepatobiliary Pancreat Dis Int. 2009 Feb; 8(1):46-9.

7. Lewis JH, Stine JG. Review article: prescribing medications in patients with cirrhosis - a practical guide. Aliment Pharmacol Ther. 2013 Jun; 37:1132

8. Verbeeck RK. Pharmacokinetics and dosage adjustment in patients with hepatic dysfunction. Eur J Clin Pharmacol. 2008 Dec;64(12):1147-61.

9. Fudeyasu K, Kawae T, Fukuhara K, et al.The effect of liver dysfunction on muscle strength in liver disease patients. Physiotherapy. 2016 Nov. 102(1): e282.

10. Zimmerman HJ, Maddrey WC. Acetaminophen (paracetamol) hepatotoxicity with regular intake of alcohol: analysis of instances of therapeutic misadventure. Hepatology. 1995 Sep; 22(3):76773

11. Kuffner EK, Dart RC, Bogdan GM, et al. Effect of maximal daily doses of acetaminophen on the liver of alcoholic patients: a randomized, doubleblind, placebo-controlled trial. Arch Intern Med. 2001 Oct; 161(18):2247-52.

12. Benson GD, Koff RS, Tolman KG. The therapeutic use of acetaminophen in patients with liver disease. Am J Ther. 2005 Mar-Apr; 12(2):133-41.

13. Rakoski M, Goyal P, Spencer-Safier M, et al. Pain management in patients with cirrhosis. Clinical Liver Disease. 2018 Jul; 11: 135-140.

14. Kirchheiner J, Schmidt H, Tzvetkov M, et al. Pharmacokinetics of codeine and its metabolite morphine in ultra-rapid metabolizers due to CYP2D6 duplication. Pharmacogenomics J. 2007 Aug; 7(4):257-65.

15. Park SH, Wackernah RC, Stimmel GL. Serotonin syndrome: is it a reason to avoid the use of tramadol with antidepressants? J Pharm Pract . 2014 Feb; 27:71-78.

16. Rogal SS, Winger D, Bielefeldt K, Szigethy E. Pain and opioid use in chronic liver disease. Digestive diseases and sciences .2013; 58(10): 2976-2985. 
17. Chandok N, Watt KD. Pain management in the cirrhotic patient: the clinical challenge. Mayo Clin Proc. 2010 May; 85(5): 451-458.

18. Imani F, Faiz HR, Sedaghat M, Hajiashrafi M. Effects of adding ketamine to fentanyl plus acetaminophen on postoperative pain by patient controlled analgesia in abdominal surgery. Anesth Pain Med. 2014 Dec;4(1):e12162.

19. Dogan S, Ozberk S, Yurci A. Pregabalin-induced hepatotoxicity. Eur J Gastroenterol Hepatol 2011 Jul; 23(7):628.

20. Jacobs JWG, Spies CM, Bijlsma JWJ , Buttgereit F. Glucocorticoids. In: Watts RA, Conaghan PG, Denton C, Foster H, Isaacs J, Müller-Ladner U, editors. Oxford textbook of rheumatology. Oxford: Oxford University Press; 2013: 607-8.

21. Donnelly S, Azeez M, MacMullan P, Whelan B. Drugs used in management of rheumatic disease. In: Callan $\mathrm{M}$, editor. The rheumatology handbook. London: Imperial College Press; 2012: 397, 406, 409-10, 412-13, 415, 417-18.
22. Talal AH, Venuto C S, Younis I. Assessment of Hepatic Impairment and Implications for Pharmacokinetics of Substance Use Treatment. Clinical pharmacology in drug development. 2017 Mar; 6(2), 206-212.

23. Terkeltaub RA, Furst DE, Bennett K, et al. High Versus Low Dosing of Oral Colchicine for Early Acute Gout Flare. Arthritis and Rheum 2010 Apr;62:1060-1068.

24. Jung Sun Lee, Jebum Won, Oh Chan Kwon, et al. Hepatic Safety of Febuxostat Compared with Allopurinol in Gout Patients with Fatty Liver Disease. The Journal of Rheumatology. 2018 Nov;jrheum.180761;DOI: https://doi.org/10.3899/jrheum.180761.

25. Khosravan R, Grabowski BA, Mayer MD, et al. The effect of mild and moderate hepatic impairment on pharmacokinetics, pharmacodynamics, and safety of febuxostat, a novel nonpurine selective inhibitor of xanthine oxidase. J Clin Pharmacol. 2006 Jan; 46(1):88102. 